\title{
BROADBAND SYNCHROTRON CAVITY FOR COSY WITH MINIMUM SIZE BASED ON VITROPERM
}

\author{
M. Böhnke, F.-J. Etzkorn, R. Maier, U. Rindfleisch, A. Schnase ${ }^{\star}$ H. Stockhorst, \\ Forschungszentrum Jülich GmbH, IKP, D-52425 Jülich
}

\begin{abstract}
A cavity design that deviates from conventional rules of RF engineering is presented. Preliminary studies in collaboration with SATURNE [1] and TERA [2] were carried out to employ the amorphous material VitroVac for a cavity with reduced size. The idea to use a nano-crystalline material instead of an amorphous one was stimulated by KEK where metglass is applied [3]. The analysis led to the magnetic material VitroPerm $500 \mathrm{~F}$, resulting in a reduced cavity size as compared to the existing one. Now, new space becomes available in COSY for additional equipment used for a polarized proton beam.

The cavity is optimized for operation at the Cooler Synchrotron COSY [4]. The frequency ranges from 400 $\mathrm{kHz}$ to $2 \mathrm{MHz}$ without any tuning device. No gapcapacitors and no cooling discs are necessary. The VitroPerm is directly water cooled. Together with the fundamental higher harmonics (2nd or 4th), adjusted in real time in amplitude and phase, can be applied to bunch or to accelerate with non-sinusoidal voltages [5].
\end{abstract}

\section{MOTIVATION OF MATERIALS DIFFERENT FROM FERRITE}

The ferrite-filled cavity being in operation at COSY is about 2.1 mlong and occupies space in one of the telescopes that will be needed for a (partial)-snake to optimize acceleration of polarized protons. The ferrite cavity has to be tuned to the actual revolution frequency and only works with sinusoidal voltages. In collaboration with SATURNE we worked on a shorter cavity $(1.4 \mathrm{~m})$ filled with the amorphous material VitroVac 6025F. This cavity operates from 0.2 to $8 \mathrm{MHz}$ and allows the application of higher harmonics together with the fundamental frequency. With such a cavity we have performed rf-gymnastics without beam to show the feasibility of the cavity for applications like:

- improved capture of injected beam

- acceleration at different harmonics

- $\gamma$-transition crossing

- stochastic cooling of bunched beams

- high power shaped noise for stochastic extraction in seconds.

However, a significantly shorter design is possible by filling another cavity with the high permeability material
VitroPerm $500 \mathrm{~F}$, shown in figure 1 . The total length is about $780 \mathrm{~mm}$, where $212 \mathrm{~mm}$ account for the vacuum gap. The 6 VitroPerm toroids per side are placed directly in water, avoiding the use of cooling plates. The broadband nature of this cavity eliminates tuning loops and allows the application of higher harmonics and noise. The impedance will be high enough to reach a maximum rfamplitude of $5 \mathrm{kV}$ (2.5 kV for each half of the structure) with an rf-power of less than $50 \mathrm{~kW}$. The disadvantages are related to the water bath: A protection against corrosion is needed, the cavity has to be protected against too high water pressure, and the purity of the water is important.

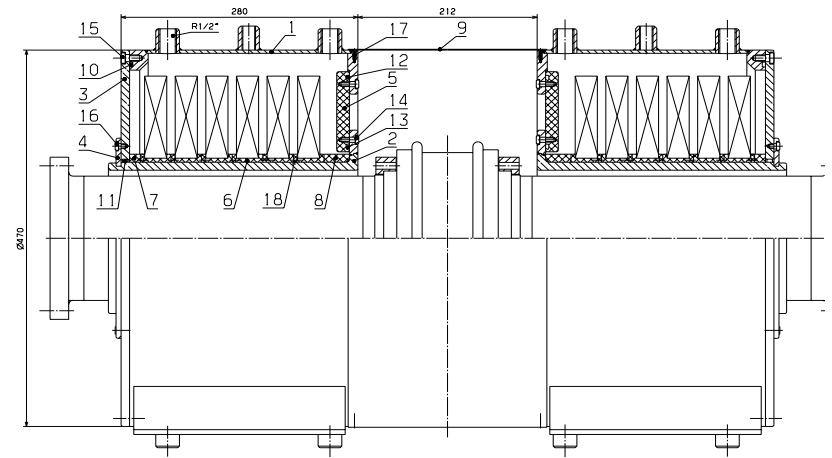

Figure 1: Layout of the broadband cavity

\subsection{Technical details of figure 1}

The housing (1) of $470 \mathrm{~mm}$ diameter is made of $5 \mathrm{~mm}$ stainless steel to stand the water pressure. The inner structure (2) is separated from the beam pipe to allow modifications without breaking the vacuum. The VitroPerm toroids are mounted on POM (DELRIN®-acetyl-resin)carriers (6) which reduces the capacitance to ground and enables high voltage operation. Circular windows (5), oriented to the gap serve as barrier between water and the air-filled outer area of the gap. The flow of the cooling water will be from bottom to top. We expect $25 \mathrm{~kW}$ to be dissipated per side, resulting in a water flow of about $201 / \mathrm{min}$.

\subsection{Measurements with samples}

We compared small toroids of VitroVac and VitroPerm. The size and the initial permeability at DC are listed in table 1 . The value for the toroids foreseen is estimated.

*email: A.Schnase@fz-juelich.de 
Table 1: Sample specifications

\begin{tabular}{|c|c|c|c|c|}
\hline & $\begin{array}{l}\text { VitroVac } \\
6025 \mathrm{~F}\end{array}$ & VitroPerm & $\begin{array}{l}\text { VitroPerm } \\
\text { W468-51 }\end{array}$ & $\begin{array}{l}\text { foreseen } \\
\text { in cavity }\end{array}$ \\
\hline sample & A & & B & estimate () \\
\hline$\emptyset_{\mathrm{i}} / \mathrm{mm}$ & 35 & 50 & 76 & 200 \\
\hline$\emptyset_{\mathrm{a}} / \mathrm{mm}$ & 72 & 80 & 102 & 400 \\
\hline $\mathrm{h} / \mathrm{mm}$ & 25 & 20 & 25 & 25 \\
\hline $\mathrm{L} / \mathrm{nH}$ & 3.336 & 1.739 & 1.361 & $(3.2)$ \\
\hline$\mu_{\mathrm{r}}$ at DC & 90000 & 30000 & 80000 & 80000 \\
\hline $\mathrm{L} / \mathrm{mH}$ at DC & 0.300 & 0.052 & 0.109 & $(0.25)$ \\
\hline
\end{tabular}

Low level impedance measurements were carried out with a network analyser HP 8751A. Figure 2a shows the impedance as a function of frequency for one VitroVac toroid (sample A) connected with a one-turn loop. This sample was tested at higher rf-power to reach temperatures of 100 ${ }^{\circ} \mathrm{C}$ in water. The coupling loop was driven by an ENIA500 amplifier. The rf-power resulting in an amplitude of $200 \mathrm{~V}$ is displayed in figure $2 \mathrm{~b}$. We observed no substantial change in impedance as temperature rises. Therefore we expect a good safety margin in operation between 20 to $50{ }^{\circ} \mathrm{C}$.

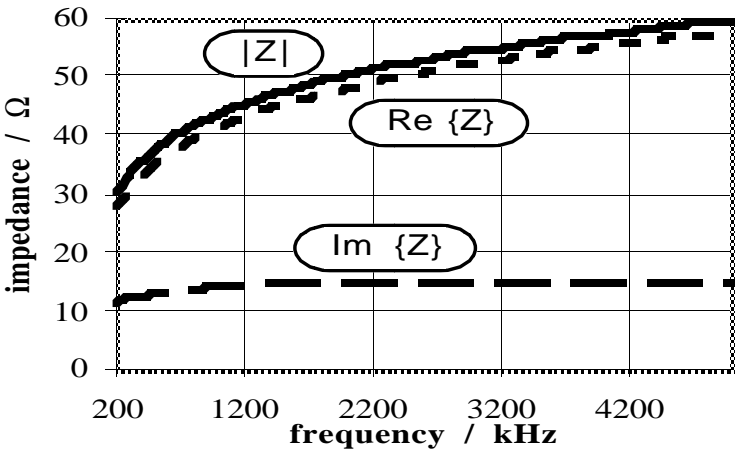

Figure 2a: Impedance measurement of VitroVac sample A

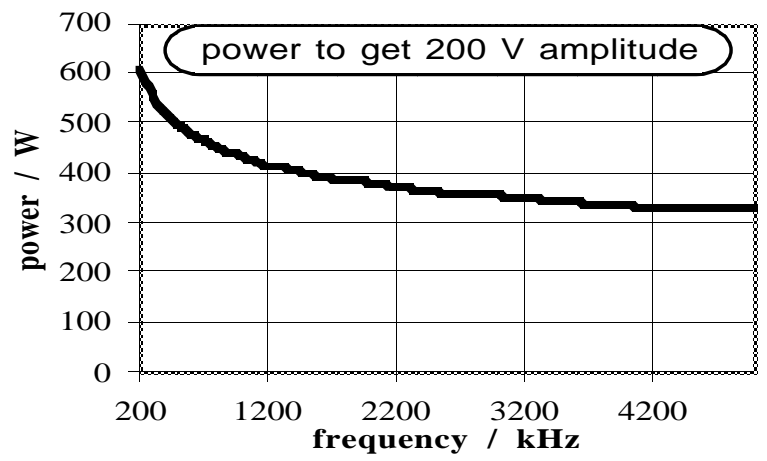

Figure 2b: Power measurement of VitroVac sample A.

In figure 3 the impedance of a combination of 4 VitroPerm sample toroids $\mathbf{B}$ in a test structure is plotted. The arrangement of 6 toroids per side in the final cavity guarantees the heat transfer to the cooling water and results in a higher impedance. The scaled result is sufficient for $2.5 \mathrm{kV}$ amplitude. The estimated power per side is displayed in figure 4.

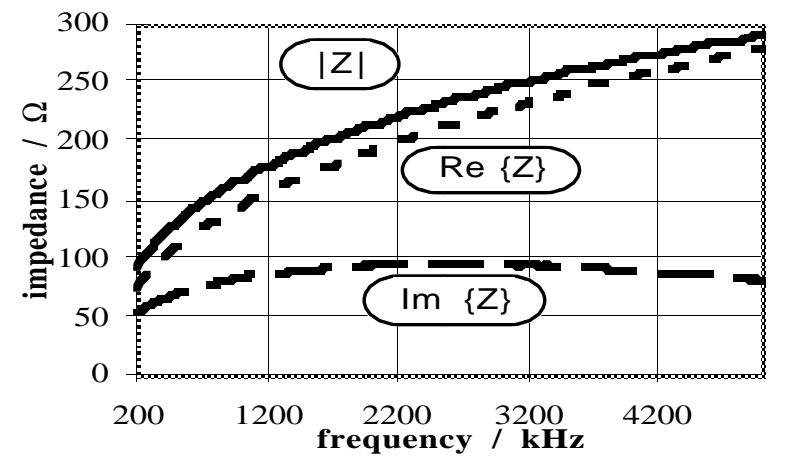

Figure 3: Impedance measurement with four samples $\mathbf{B}$.

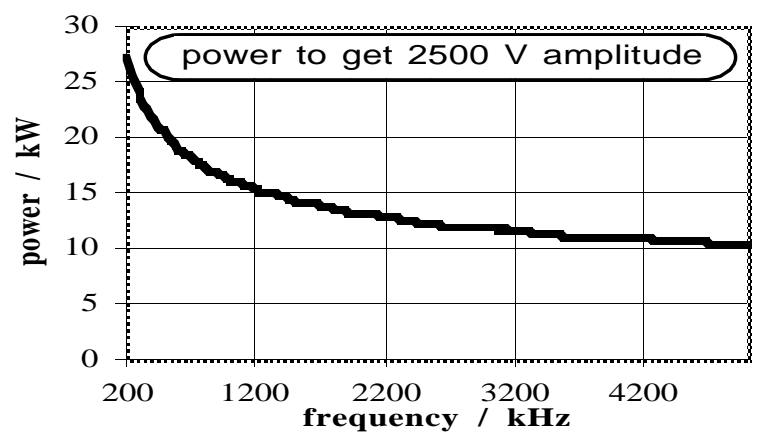

Figure 4: Estimated power from impedance measurement.

\subsection{Application of higher harmonics}

The suitability of the cavity for higher harmonics is proven by a setup where a signal composed of a fundamental at $500 \mathrm{kHz}$ and two harmonics drives an amplifier AR 15A250 directly connected to the teststructure with four toroids. The broadband behavior is clearly visible in figure 5: the upper trace is the predistorted generator signal and the lower trace the voltage at the structure measured with a 1:10 probe.

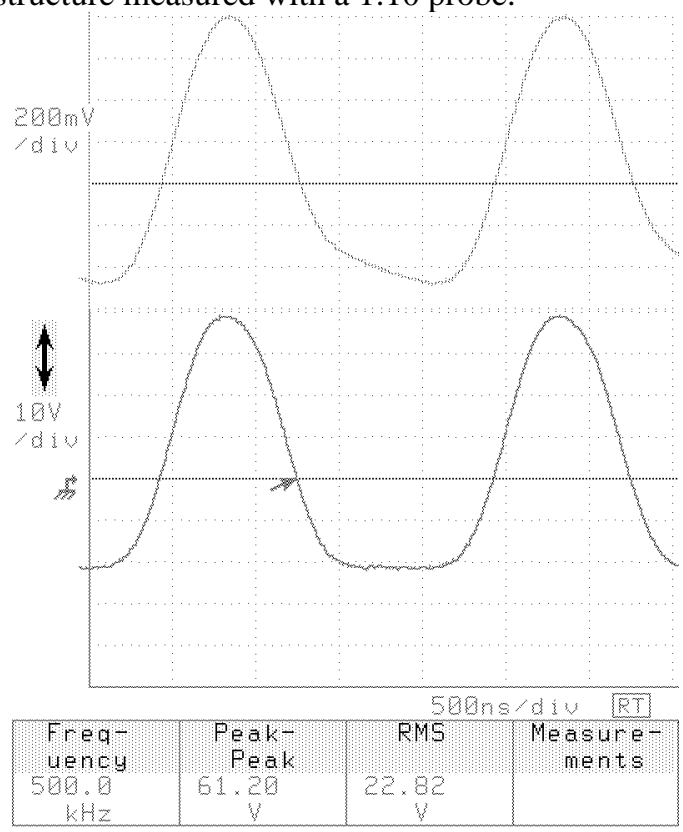

Figure 5: Waveform with a flat top, power $\approx 4 \mathrm{~W}$. 


\section{SIGNAL GENERATION}

A dedicated synthesizer [6] generates the higher harmonics for the measurement of the behavior of the VitroPerm in time domain. It has been upgraded to perform a synchronous sweep with the nonlinear frequency ramp of COSY while preserving the shape of the waveform. The Fourier components are controlled in real time. Figure 6 depicts the structure of signal generation. The hardware is installed in 2 VME-crates. One of the crates contains a communication CPU and 7 DSP boards which generate the digital set values of the different harmonics as a function of time. The amplitude is controlled with a DAC that drives an analog multiplier. The frequency word is fed to a numerical oscillator (NCO) that generates the clock $16 \cdot f_{\text {rev }}$ for the Lookup-tables, the 16 bit complex multipliers and the DAC, where $\mathrm{f}_{\text {rev }}$ varies between $0.4 \mathrm{MHz}$ to 1.6 MHz. The amplitudes of the higher harmonics are much lower than the fundamental. Therefore the signal of the DACs is fed to attenuators, to keep the signal-to-noise ratio of $12 \ldots 14$ bit, which results in $-70 \mathrm{dBc}$ when measured.

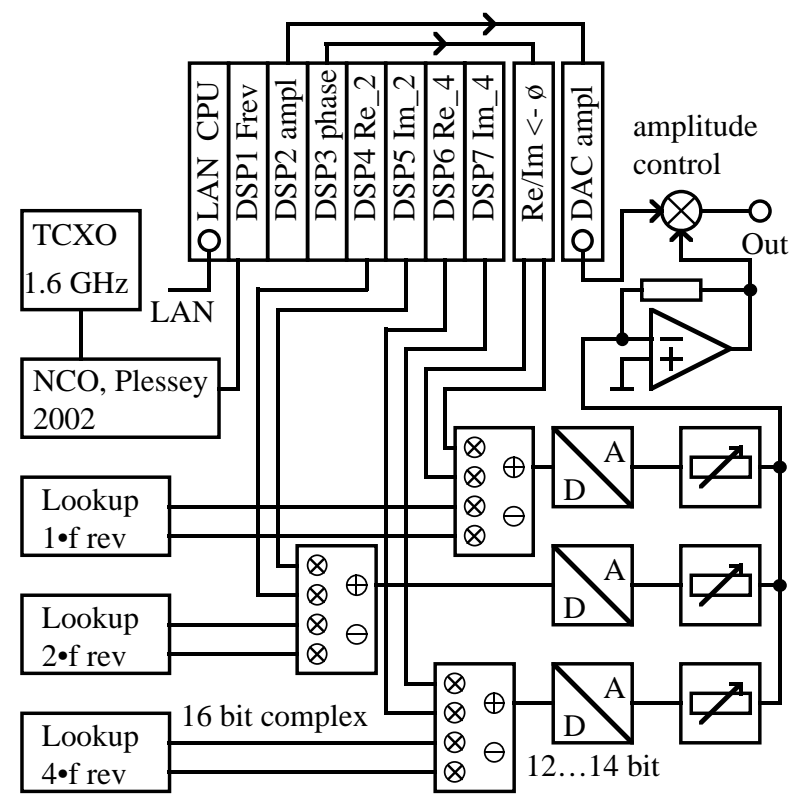

Figure 6: Layout of agile signal synthesis

\section{INSTALLATION PROCEDURE}

The preparation of the installation of the cavity is on its way. In the end of June 99 we expect to have the structure and the material available. We will test the cavity performance at low level and stress the structure to find out the influence of water pressure. We will find out, if the impedance, influenced by the capacitance of the water - some $100 \mathrm{pF}$ - is high enough, or if we have to rearrange the position of the cores inside the water tank.

Due to the compact size and small weight of the cavity and the material, the setup is mobile. We therefore plan to perform first power measurements at the test bed of the VitroVac cavity at CERN. When we know the power requirements $(50 \ldots 100 \mathrm{kVA})$, we will install the tube amplifier in the "eye" of the COSY-ring. The anode power supply will be specified to minimize operational cost. In the mean time we use the anode power supply of the existing tube amplifier to feed the amplifier of the broadband structure. If the new cavity performs as expected, we install it in the target telescope in a way that allows to switch between the ferrite cavity and the higher harmonic cavity in reasonable time. A Klöckner-Möller SPS will control the power supplies and check for error and fault conditions. The locations of the existing ferrite cavity and the new one in COSY are sketched in figure 7.

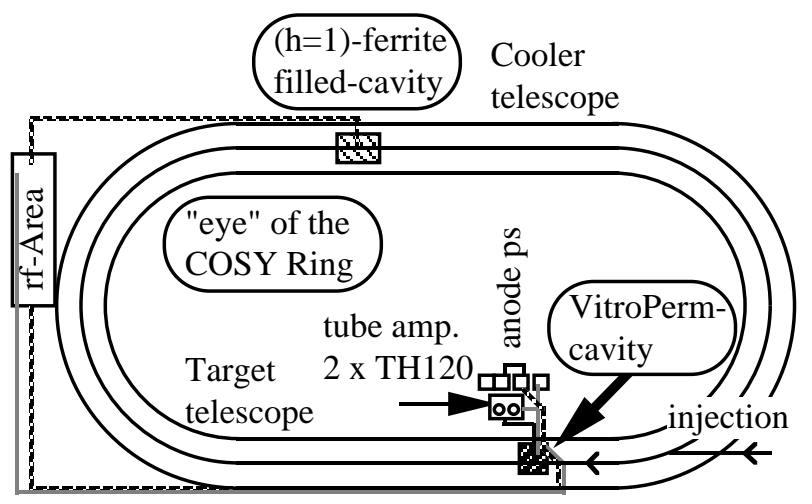

Figure 7: Locations of the rf-systems in the COSY-ring.

\section{OUTLOOK}

An interesting question will be the connection of the amplifier to the cavity gap. Depending on the installation, a distance of 3 to $7 \mathrm{~m}$ has to be bridged with an coaxial air filled transmission line of $200 \Omega$ impedance.

With both cavities operational this year, we have new opportunities in modifying the longitudinal phase-space. We will find out, if the cavity will perform as expected to replace the existing one, which then acts as a backup.

\section{REFERENCES}

[1] P. Ausset et. al. „A Broad-Band $(0.2-8 \mathrm{MHz})$ MultipleHarmonic VITROVAC®-Filled Acceleration Structure“, EPAC94, Berlin

[2] A. Schnase et. al. „Higher Harmonic (VitroVac) Cavity: Measurement results with the Prototype“, IKP Annual report 1997, Forschungszentrum Jülich GmbH

[3] Y. Mori et al. „A new type of RF cavity for high intensity proton synchrotron using high permeability magnetic alloy“, EPAC98, Stockholm

[4] H. Stockhorst et. al. „The Performance Of COSY“, EPAC 98, Stockholm

[5] H. Stockhorst ,Longitudinal Beam Dynamics in a Multiple Harmonic RF-System“, IKP Annual report 1994, Forschungszentrum Jülich $\mathrm{GmbH}$

[6] F.-J. Etzkorn et. al. „Towards a cavity for higher harmonics“, IKP Annual report 1994, Forschungszentrum Jülich GmbH 